\title{
La Guía Docente: un reto en el nuevo modelo de educación universitaria
}

\author{
Emilio C. GARCía FernÁNDEZ \\ Universidad Complutense de Madrid \\ avia@ccinf.ucm.es \\ Luis DeLTell Escolar \\ Universidad Complutense de Madrid \\ ldeltell@ccinf.ucm.es
}

Recibido: 24/07/2012

Aceptado: 26/10/2012

\begin{abstract}
Resumen
La incorporación al Espacio Europeo de Educación Superior (EEES) conlleva una nueva dinámica en la fundamental para esta nueva relación entre docente y discente. cias de la Información en la Universidad Complutense de Madrid. mente al EEES.

Palabras clave: enseñanza, aprendizaje, EEES, Guía Docente, comunicación

\section{Syllabus (Guía Docente): a Challange in the New Model Education at University Level} enseñanza-aprendizaje. Los nuevos modelos educativos exigen una nueva forma de organizar y presentar las asignaturas. En este artículo investigamos sobre el uso de la Guía Docente como una herramienta

Esta investigación se basa en una serie de entrevistas a profesores, en la ayuda y elaboración de Guía Docentes y en el estudio de su aplicación en el Grado de Comunicación Audiovisual en la Facultad de Cien-

Proponemos un cambio en el uso de la Guía Docente que se aleja del viejo programa para educar plena-

\begin{abstract}
The transition to the European Higher Education Area (EHEA) requires a new teaching-learning paradigm. This educational model demands a different way to organize and present the subject material. In this paper we research the use of a Syllabus (Guía Docente) as a fundamental tool for this relationship between the professor and the student.

This study is based o a series of interviews with professors and the analysis of Syllabus in the Communication Degree (Facultad de Ciencias de la Información) at the Complutense University in Madrid (Universidad Complutense de Madrid).

We propose a change in the current teaching methodology, which will be reflected in the EHEA Syllabus. Keywords: Teaching, Learnig, EHEA, Syllabus, Communication

\section{Referencia normalizada}

GARCÍA FERNÁNDEZ, Emilio C. y DELTELL ESCOLAR, Luis (2012): "La Guía Docente: un reto en el nuevo modelo de educación universitaria". Estudios sobre el mensaje periodístico. Vol. 18, núm. especial octubre, págs.: 357-364. Madrid, Servicio de Publicaciones de la Universidad Complutense.
\end{abstract}

Sumario: 1. Introducción, fuentes y estado de la cuestión. 2. Metodología y campo de estudio. 3. Desarrollo; 3.1. Datos básicos; 3.2. Denominación en el Grado; 3.3. Sobre el sistema de evaluación; 3.4. Metodología de enseñanza y aprendizaje; 3.5. Sobre los créditos ECTS; 3.6. El Campus Virtual y apoyo en nuevas tecnologías; 3.7. El programa de la asignatura. Los contenidos; 3.8. La tutoría; 3.9. Bibliografía y documentos de consulta. 4. Conclusión. 5. Referencias bibliográficas.

\section{Introducción, fuentes y estado de la cuestión}

El proyecto educativo que se conoce como Espacio Europeo de Educación Superior (EEES) y la Declaración de Bolonia, surgida de la reunión mantenida en Bolonia el 
19 de junio de 1999 por los Ministros Europeos de Educación, se asientan, especialmente, sobre dos pilares insustituibles: el profesor y el alumno. No se trata de que uno camine en paralelo al otro, sino que el nuevo modelo obliga a un trabajo en común durante el tiempo que dure la permanencia del estudiante en la Universidad.

El alumno asume una gran responsabilidad en su formación. Ya no puede ser la persona pasiva que se conforma con tomar notas de lo que explica el profesor, sino que ha de participar activamente en su itinerario formativo para alcanzar los objetivos propuestos. El docente, por su parte, se convierte en el tutor, asesor, la ayuda imprescindible para el alumno, su sombra, porque es quien orientará adecuadamente al alumno en cada uno de sus pasos. En palabras de la Comisión Técnica del Ministerio de Educación y Ciencia: "Cada día más, la enseñanza universitaria tendrá que responder a situaciones de enseñanza-aprendizaje diversas, que abarcan desde las más convencionales y tradicionales, hasta las más novedosas [...] Se requiere, en definitiva, introducir en nuestro nivel de enseñanza otro estilo" (Ministerio de Educación y Ciencia, Comisión Técnica, 2007: 63).

Pero el papel que debe asumir cada uno de los protagonistas del Espacio Europeo de Educación Superior todavía no se ha entendido bien. Y en algunos casos ha sido duramente criticado (Llovet, 2011: 189). Son muchos los problemas que se alegan para el cumplimiento de las líneas generales que surgen del EEES. Los profesores exponen continuamente la masificación de las aulas, la impotencia para desarrollar un modelo tutorial personalizado y la pasividad de los alumnos como algunos de estos óbices.

La experiencia de dos años en el desarrollo del EEES nos permite conocer muchos de los problemas generados en la implantación del nuevo modelo educativo, al tiempo que nos ha obligado a implantar fórmulas diferentes y complementarias que nos han ayudado a entender mucho mejor el modelo y aceptar que parte del trabajo del docente es, en palabras de Ken Bain (2007: 1995), "reformular lo que significa ser profesor". Sin duda, la herramienta más adecuada para la integración de esta nueva formulación es la Guía Docente, eje vertebral del modelo formativo a desarrollar.

En la actualidad se han publicado artículos y trabajos sobre el uso de las Guías Docentes en los grados de Música (Gil Asensio, 2008: 17-27), Publicidad (Fanjul Peyró, 2010: 82-85), en carreras del área de Salud (Morón Marchena y Pedrero García, 2012: 375-390) y otras. Consideramos relevante introducir en este debate científico también en el campo de los estudios de la Comunicación Audiovisual y, muy en concreto, los estudios de Historia de la imagen y del cine, asignaturas entendidas tradicionalmente como teóricas.

En este texto planteamos el uso de la Guía Docente para organizar y entender la práctica de la enseñanza-aprendizaje en el EEES. No solo defendemos su utilidad sino que, además, proponemos un uso del mismo atendiendo a los errores y a la experiencia de los dos primeros años de aplicación de grado en Comunicación Audiovisual en la Facultad de Ciencias de la Información de la Universidad Complutense de Madrid.

\section{Metodología y campo de estudio}

Nuestro estudio se basa en el análisis y uso que se debe hacer de la Guía Docente, equivalente en gran medida al "Syllabus" contemporáneo de las universidades norte- 
americanas (Fink, 2003: 67). Para ello hemos seguido y analizado las Guías Docentes en el área de Comunicación Audiovisual y, en concreto, en las asignaturas de Historia General de la Imagen, Historia del Cine Español e Historia del Cine.

Aún hoy, la Guía Docente no se encuentra bien definida y se confunde con frecuencia con un programa o con un calendario de actividades básicas. En realidad, la Guía Docente es el contrato que asumen profesor y alumno durante el curso. Debe ser un texto que permita seguir los pasos de los contenidos, el trabajo personal que ha de ir realizando el alumno y cómo ha de participar activamente en cada una de las fases del proceso formativo. El alumno debe leer a fondo todo lo que se dice en dicha Guía Docente para entender claramente qué es lo que ofrece el profesor y qué se le pide a lo largo del curso. En nuestra metodología nos centramos también en abordar el problema de la exigencia mutua entre alumno y profesor. Así como el uso de las tutorías personalizadas y de enseñanza individualizada para cada alumno.

Para realizar este estudio hemos utilizado una metodología cualitativa basada entrevistas a profesores del área siguiendo el modelo de Ken Bain (2007: 201) y Mihaly Csikszentmihalyi (2011: 22). En dicha entrevista preguntamos sobre cómo desarrollan la asignatura y la reflejan en la Guía Docente y en qué medida obtienen resultados válidos en cada curso. Nuestro trabajo se campo se ha extendido a varias Universidades españolas en donde se imparten las materias y a una veintena de profesores a los cuales se les ha sometido a entrevistas en profundidad. Además hemos colaborado en la elaboración (y su posterior verificación) de una docena de Guías docentes en el campo de la Comunicación Audiovisual y la Publicidad en la Facultad de Ciencias de la Información de la Universidad Complutense de Madrid.

Así mismo hemos combinado esta metodología cualitativa con un seguimiento cuantitativo de las Guía Docentes de dicho centro basándonos en el modelo de enseñanza propuesto por la Secretaría de Estado (MINISTERIO DE EDUCACIÓN Y CIENCIA, Comisión Técnica, 2007: 9-11). En este estudio se han analizado un total de treinta Programas de asignaturas y Guías Docentes directamente vinculadas al área de Comunicación Audiovisual, Publicidad y de Periodismo. En ellas se han estudiado aspectos de: a) contenidos y temarios; b) información de sistema evaluación; c) tutorías; d) garantías del estudiante; e) horarios; f) prácticas y trabajos de laboratorios; y g) uso de Campus Virtual y e-docencia.

El análisis de los datos conseguidos con esta metodología, y nuestra experiencia anual, nos ha permitido elaborar un nuevo modelo de Guía Docente.

\section{Desarrollo}

A partir de las entrevistas y de los datos obtenidos hemos esbozado un análisis de los contenidos que deben estar recogidos en la Guía Docente de cada Grado, que previamente, como es sabido, ha debido ser aprobado por cada Centro, Universidad y verificada por la Agencia Nacional de Evaluación de la Calidad y Acreditación (ANECA), entre otras instituciones.

Los aspectos fundamentales de la Guía Docente los hemos abordado en nueve puntos: 1 . Datos básicos; 2. Denominación en el Grado; 3. Sobre el sistema de evaluación; 4. Metodología de enseñanza y aprendizaje; 5. Sobre los créditos ECTS; 6. El Cam- 
pus Virtual y apoyo en últimas tecnologías; 7. El programa de la asignatura. Los contenidos; 8. La tutoría; 9. Bibliografía y documentos de consulta.

Es decir, lo que se pretende es que "el diseño de la Guía Docente (vaya) más allá de la tradicional programación didáctica de la asignatura. Supone el establecimiento de un marco de referencia útil" (Gil Asensio, 2008: 27).

\subsection{Datos básicos}

El profesor debe recoger en su Guía Docente todos los datos identificadores de la asignatura para que el alumno pueda acceder fácilmente a la información complementaria. Es así que debe reseñar el carácter de la misma -si es obligatoria u optativa-, los horarios de clase, el despacho en el que atenderá a los alumnos y el horario de tutorías.

Y debe ser razonable que el alumno tenga referencias concretas del profesor, que conozca su currículum -que siempre lo tendrá al alcance de la Web institucional- y sepa con qué recursos docentes cuenta. Esta información le ayudará para hablar con el enseñante, para saber qué preguntas hacerle y concretar aspectos de la materia que imparte. Este conocimiento ayudará en un mejor desarrollo de la relación entre profesor y alumno.

\subsection{Denominación en el Grado}

Para comprender qué es un Grado, debe entenderse que una asignatura forma parte de una materia y esta de un módulo más amplio; es decir, un módulo puede encerrar varias materias que, a su vez, engloben varias asignaturas que en su conjunto -en un curso o en varios- proporcionan al alumno unos conocimientos amplios sobre contenidos que son fundamentales para su formación. Esto debe considerarse como un sistema organizado de elementos que permitirán que la improvisación no afecte a lo sustancial y que todo tenga su sentido en la estructura formativa que se ha diseñado.

\subsection{Sobre el sistema de evaluación}

En este caso hay que partir del número de alumnos por grupo. Cada carrera tiene su propio diseño y de su especificidad depende el ingreso de más o menos alumnos. Está claro que grupos reducidos permiten desarrollar una evaluación continua sin ningún problema, porque el profesor podrá conocerlos personalmente y los alumnos mostrarán una mayor predisposición a un seguimiento diario. Sin embargo, en aquellas carreras más masificadas, hablar de grupos con un mínimo de noventa alumnos supone establecer otros criterios que deben combinar varias fórmulas para poder aplicar un sistema adecuado de evaluación de resultados; en este caso, los alumnos estarán más desamparados que los anteriores y sujetos a otros elementos de juicio que el profesor tendrá que aplicar.

En cualquier caso, la evaluación debe regirse por "utilizar la calificación para ayudar a los estudiantes a aprender, no sólo para clasificar y jerarquizar su esfuerzos" (Bain, 2007: 169). Por tanto, la evaluación continua exige, sin duda, la participación activa y permanente del alumno. Obliga al estudiante, siempre, a preparar a la par que el profesor los temas que se exponen en el aula, a reflexionar y discutir sobre ellos y argumentar sugerencias que complementen lo explicado. 
Por eso, el seguimiento diario, la tutoría personalizada y aquellos recursos que el profesor pone al alcance del alumno durante el curso construyen el edificio formativo sobre el que se asentarán los conocimientos adquiridos.

\subsection{Metodología de enseñanza y aprendizaje}

Un punto esencial de la Guía Docente es el que recoge todo lo relativo a las actividades formativas (con su contenido en créditos ECTS), la metodología de enseñanzaaprendizaje y su relación con las competencias que debe adquirir el estudiante.

Después de varios métodos estudiados, hemos llegado a la conclusión de que una herramienta formativa ideal es aquella que obliga al alumno a esforzarse en la preparación de un tema de la materia impartida. Sin invertir la relación docente-discente se consigue que el alumno se forme a sí mismo elaborando parte del temario y que "sean capaces de inventar en lugar de limitarse a repetir" (González Soto y Sánchez Delgado, 2005: 17). Tres son las consecuencias positivas de dicha tarea: La primera, que el alumno se siente parte fundamental de la tarea educativa. La segunda, que el alumno aprende a utilizar fuentes documentales de todo tipo. Es evidente que el aula, el entorno enseñanza-aprendizaje, debe integrar muchas de las novedades que se han asentado en la vida diaria de los alumnos. Y, la tercera consecuencia, la aportación personal que proporciona al alumno un sentimiento de seguridad en el proyecto.

\subsection{Sobre los créditos ECTS}

De acuerdo con el Informe sobre el Sistema Europeo de Transferencia y Acumulación de Créditos (ECTS- European Credit Transfer System): "el ECTS se basa en la convención de que 60 créditos miden la carga de trabajo de un estudiante a tiempo completo durante un curso académico. La carga de trabajo para un estudiante en un programa de estudios a tiempo completo en Europa equivale, en la mayoría de los casos, a 1.500 o 1.800 horas por año, y en tales casos un crédito representa de 25 a 30 horas de trabajo" (VV.AA., 2004: 4). El acuerdo final es de un crédito igual a 25 horas.

Esta disposición ejemplifica cómo ha de distribuir el profesor la carga docente de la asignatura y de qué manera ha de implicarse el alumno en desarrollar personalmente todo aquello que le competa directamente.

Los créditos son, sin duda, una herramienta para poder ir viendo cómo avanza el alumno y si está en condiciones de superar la asignatura una vez realizado todo lo programado por el profesor. Como dice el Informe arriba recogido: "La carga de trabajo del estudiante en el ECTS consiste en el tiempo invertido en asistencia a clases, seminarios, estudio personal, preparación y realización de exámenes, etc. " (VV.AA., 2004: 4). La nota final saldrá de los porcentajes aplicados por el profesor en cada una de las partes de la asignatura.

\subsection{El Campus Virtual y apoyo en nuevas tecnologías}

El EEES obliga al docente a modificar parte del sistema de educación. Uno de los aspectos necesarios es la creación de un espacio Web en el Campus Virtual de su Universidad o en otros entornos digitales que le permitan ofrecen contenidos sobre su materia a los alumnos. Estos deben regirse por algunos parámetros esenciales. Estas herramientas tecnológicas deben ser entendidas como "soluciones de mayor flexibi- 
lidad, que potencian un modelo de instrucción más individualizado y personalizado" (Hernando Sanz, 2005: 218). En el caso concreto del grado de Comunicación Audiovisual el uso de las tecnologías más recientes deja de ser una herramienta de trabajo para ser una competencia básica en la formación del alumno.

Uno de los peligros de las nuevas tecnologías es el uso indebido por parte del discente. En especial "el copy-paste y el plagio infinito" (Deltell, 2007: 310) por medio de buscadores de información. Sin embargo y a pesar de estos comportamientos fraudulentos, el uso del Campus Virtual y la tecnología resulta esencial en la formación del alumno.

\subsection{El programa de la asignatura. Los contenidos}

El alumno debe conocer desde el primer día cuáles son los contenidos que el profesor ha preparado para desarrollar su asignatura a lo largo del curso. El temario le debe servir de guía para ir adquiriendo los conocimientos propuestos como básicos y generales. Está claro que los temas y los subtemas deben ser los que orienten al alumno para leer sobre los mismos y ampliar todo aquello que se ha dicho en el aula. El profesor ha de reflejar de manera precisa todos los temas a desarrollar, con el fin de que el alumno no tenga ninguna duda.

\subsection{La tutoría}

El profesor tiene la obligación de indicar en la Guía Docente cuáles son sus horarios de atención al alumno. Dichos horarios se hacen públicos no para cumplir un mandato académico sino para que el alumno sepa cuando puede consultar al profesor.

La tutoría es el lugar y el espacio donde la "función docente" debe pasar a convertirse en una "función tutorial" de guía (Álvarez González, 2007: 85-86). Por ello, el profesor debe reflejar no solo los horarios de la misma, sino también las actividades y corrección de trabajos que se proponen para esas horas.

\subsection{Bibliografía y documentos de consulta}

En la Guía Docente, el profesor debe reflejar, detalladamente, la bibliografía principal y complementaria que ha de utilizar el alumno para completar su formación. Esta idea debe transmitirse desde el primer día de clase. El alumno no puede limitarse a lo comentado por el profesor en el aula. El profesor ofrece en su exposición unas directrices que el alumno ha de ampliar con sus lecturas.

A pesar la discusión teórica sobre el futuro de la bibliografía y de su uso (Capaccioni, 2008: 105-109), la Guía Docente debe recogerla y el docente debe invitar a su manejo.

La bibliografía se deben dividir en obras fundamentales (manual), básicas y complementarias. Siguiendo el siguiente esquema: a) Referencia fundamental o manual (obligatoria lectura en su totalidad); b) Referencia básica (fragmentos de obligatoria lectura y lecturas recomendadas); y c) Referencia complementaria y de apoyo (siempre recomendada y en ningún caso obligatoria).

\section{Conclusión}

Para entender el nuevo modelo de educación universitaria estamos convencidos de que se debe asumir el compromiso del uso de la Guía Docente. Tres son los aspectos esenciales: 
1. Que los profesores deben contemplar la Guía Docente como la mejor herramienta de orientación para que el alumno sepa hacer frente a la formación que se le proporciona en una determinada asignatura.

2. Que el alumno debe asumir su protagonismo en el ciclo formativo y entender el manejo de dicho documento

3. Que docente y discente tienen que asumir que la formación se reparte entre ambos a lo largo del curso académico y que la responsabilidad de que los objetivos se alcancen, también es de los dos.

Con todo esto llegamos a un punto de encuentro que se debe proyectar en la Evaluación y Autoevaluación vinculadas a los programas de evaluación del profesorado (Programa Docentia). Los estudios y análisis de evaluación que se aplican al docente deben incluir un estudio de las Guías Docentes y del manejo de las mismas.

El reto del EEES supone un nuevo cambio de paradigma en la enseñanza-aprendizaje. El profesor y el estudiante se encuentran con una situación nueva en la cual los viejos modelos han quedado obsoletos. Los programas, los temarios y los resúmenes de asignatura deben ser sustituidos por Guías Docentes que se adecuen a la nueva realidad y a las nuevas propuestas.

\section{Referencias bibliográficas}

ALVÁREZ GONZÁLEZ, Manuel (2008): "La tutoría académica en el espacio europeo de la Educación Superior". Revista interuniversitaria de formación del profesorado, $\mathrm{n}^{\mathrm{o}}$ 61. Zaragoza, Asociación Universitaria de Formación del Profesorado, pp. 71-88.

BAIN, Ken (2007): Lo que hacen los mejores profesores universitarios. Valencia, PUV.

CAPACCIONI, Andrea (2008): "El papel y el futuro de la Bibliografía". Documentación de las Ciencias de la Información, $\mathrm{n}^{\circ}$ 31. Madrid, Servicio de Publicaciones de la Universidad Complutense, pp. 105-109.

CHAMORRO PLAZA, María del Carmen y SÁNCHEZ DELGADO, P. (Coords., 2005): Iniciación a la docencia universitaria. Manual de ayuda. Madrid, ICE de la Universidad Complutense.

CSIKSZENTMIHALYI, Mihalyi (2011): Creatividad. El fluir y la psicología del descubrimiento y de la invención, Barcelona, Paidós, Transiciones.

DELTELL, Luis: "El plagio infinito. Estudio sobre el plagio digital en la enseñanza universitaria" en WALZER, Alejandra, GARCÍA LÓPEZ, Marcial y RODRÍGUEZ, Juan Carlos (2007): Comunicación alternativa, ciudadanía y cultura, Madrid, Foro Universitario, pp. 308-314.

FANJUL PEYRÓ, Carlos y otros: "La guía docente como herramienta activa en la praxis didáctica de materia de Publicidad y RR.PP. adaptadas al EEES", Revista d'innovació educativa, $\mathrm{n}^{\circ}$ 4, Valencia, Universitat de Valencia, pp. 83-85.

FINK, Dee (2003): Creating Significant Learning Experiences. San Francisco, JosseyBass. 
GILASENSIO, Vicent (2008): "La guía docente: punto de partida para la concreción de un nuevo grado superior de música adaptado al EEES", Revista electrónica de LEEME, $\mathrm{n}^{\circ}$ 21, Valencia, Universitat de Valencia, pp. 17-27.

GONZÁLEZ SOTO, Ángel Pío y SÁNCHEZ DELGADO, Primitivo (2005): “¿Qué sabes de cómo aprenden nuestros alumnos en la Universidad? Proceso de aprendizaje adulto", en CHAMARRO PLAZA, María del Carmen y SÁNCHEZ DELGADO, Primitivo (Coord.): Iniciación a la docencia universitaria. Manual de ayuda, Madrid, Universidad Complutense de Madrid, Instituto de Ciencias de la Educación.

HERNANDO SANZ, Felipe (2005): "El uso de las nuevas tecnologías en el aula universitaria en el marco de las EEES", en CHAMARRO PLAZA, María del Carmen y SÁNCHEZ DELGADO, Primitivo (Coord.): Iniciación a la docencia universitaria. Manual de ayuda, Madrid, Universidad Complutense de Madrid, Instituto de Ciencias de la Educación.

LLOVET, Jordi (2011): Adiós a la universidad. El eclipse de las Humanidades. Barcelona, Galaxia Gutenberg, Círculo de Lectores.

MINISTERIO DE EDUCACIÓNY CIENCIA(2006): Propuestas para la renovación de las metodologías educativas en la Universidad. Madrid, Secretaría General Técnica.

MORÓN MARCHENA, Juan Agustín y PEDRERO GARCÍA, Encarnación (2012): "Orientando el proceso de enseñanza-aprendizaje en la asignatura de educación para la salud: la guía docente en el espacio europeo de educación superior", Revista científica de la Universidad Pablo Olavide, Sevilla, Universidad de Pablo Olavide, $\mathrm{n}^{\mathrm{o}} 1$, pp. 375-390.

VV.AA. (2004): Informe sobre el Sistema Europeo de Transferencia y Acumulación de Créditos (ECTS- European Credit Transfer System), Luxemburgo, Oficina de Publicaciones Oficiales de las Comunidades Europeas.

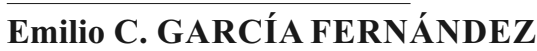

Catedrático de Universidad avia@ccinf.ucm.es Universidad Complutense de Madrid.

Facultad de Ciencias de la Información.

Departamento de Comunicación Audiovisual y Publicidad 1

Avenida Complutense s/n. 28040. Madrid

\section{Luis DELTELL ESCOLAR}

Profesor Contratado Doctor.

ldeltell@ccinf.ucm.es

Universidad Complutense de Madrid.

Facultad de Ciencias de la Información.

Departamento de Comunicación Audiovisual y Publicidad 1

Avenida Complutense s/n. 28040. Madrid 